\title{
Resveratrol Derivative-Rich Melinjo Seed Extract Attenuates Skin Atrophy in Sod1-Deficient Mice
}

\author{
Kenji Watanabe, Shuichi Shibuya, Yusuke Ozawa, Naotaka Izuo, and Takahiko Shimizu \\ Department of Advanced Aging Medicine, Chiba University Graduate School of Medicine, Chiba 260-8670, Japan \\ Correspondence should be addressed to Takahiko Shimizu; shimizut@chiba-u.jp
}

Received 24 September 2014; Accepted 18 November 2014

Academic Editor: Dragan Milenkovic

Copyright (c) 2015 Kenji Watanabe et al. This is an open access article distributed under the Creative Commons Attribution License, which permits unrestricted use, distribution, and reproduction in any medium, provided the original work is properly cited.

\begin{abstract}
The oxidative damages induced by a redox imbalance cause age-related changes in cells and tissues. Superoxide dismutase (SOD) enzymes play a pivotal role in the antioxidant system and they also catalyze superoxide radicals. Since the loss of cytoplasmic SOD (SOD1) resulted in aging-like phenotypes in several types of murine tissue, SOD1 is essential for the maintenance of tissue homeostasis. Melinjo (Gnetum gnemon Linn) seed extract (MSE) contains trans-resveratrol (RSV) and resveratrol derivatives, including gnetin $\mathrm{C}$, gnemonoside A, and gnemonoside D. MSE intake also exerts no adverse events in human study. In the present studies, we investigated protective effects of MSE on age-related skin pathologies in mice. Orally MSE and RSV treatment reversed the skin thinning associated with increased oxidative damage in the Sod1 ${ }^{-1-}$ mice. Furthermore, MSE and RSV normalized gene expression of Colla1 and p53 and upregulated gene expression of Sirt1 in skin tissues. In vitro experiments revealed that RSV significantly promoted the viability of Sod1 ${ }^{-1-}$ fibroblasts. These finding demonstrated that RSV in MSE stably suppressed an intrinsic superoxide generation in vivo and in vitro leading to protecting skin damages. RSV derivative-rich MSE may be a powerful food of treatment for age-related skin diseases caused by oxidative damages.
\end{abstract}

\section{Introduction}

Intrinsic skin aging induced by chronological or intrinsic factors leads to skin atrophy [1]. Skin collagen components show age-dependent reductions in both male and female subjects, resulting in age-related skin thinning in older individuals [2]. Accumulated evidence suggests that oxidatively modified proteins, DNA, and lipids in the skin and other organs during aging are progressively accumulated [3], indicating that reactive oxygen species (ROS) are strongly associated with skin aging. To attenuate oxidative damages, multiple antioxidative and repair systems exert in cells. Superoxide dismutase (SOD) plays a central role in the antioxidative systems due to its ability to catalyze cellular superoxide radicals $\left(\mathrm{O}_{2}{ }^{-{ }^{-}}\right)$to $\mathrm{H}_{2} \mathrm{O}_{2} \cdot \mathrm{H}_{2} \mathrm{O}_{2}$ is further degraded to $\mathrm{O}_{2}$ and $\mathrm{H}_{2} \mathrm{O}$ by catalase, glutathione peroxidases, and peroxiredoxins. Copper/zinc superoxide dismutase (SOD1) is localized to react intracellular $\mathrm{O}_{2}{ }^{--}$in the cytoplasm. Our previous studies demonstrated that Sodl-deficient $\left(\mathrm{Sodl}^{-/-}\right)$mice showed enhancement of intracellular $\mathrm{O}_{2}{ }^{--}$and various aging-like organ phenotypes, suggesting that cytoplasmic $\mathrm{O}_{2}{ }^{--}$-mediated oxidative damages primarily cause aging-like changes in various tissues [4]. Particularly, Sod1 insufficiency resulted in both epidermal and dermal atrophies associated with downregulation of extracellular matrix-related genes including Collal and with upregulation of age-related genes including p53 $[5,6]$. Therefore, $\mathrm{Sodl}^{-/-}$mouse is a suitable model for studying skin aging in older people.

Melinjo (Indonesian name; Gnetum gnemon Linn) is an arboreal dioecious plant that is widely cultivated in Southeast Asia. Its fruits and seeds are used as an ordinary vegetable in Indonesia. Melinjo seeds contain various stilbenoids including trans-resveratrol $\left(3,5,4^{\prime}\right.$-trihydroxy-trans-stilbene), its glucoside, resveratrol dimer (gnetin C), and resveratrol dimer glucoside (gnetin L, gnemonoside $\mathrm{A}$, gnemonoside $\mathrm{C}$, and gnemonoside D) [7]. Melinjo seed extract (MSE) revealed DPPH radical scavenging [7], lipase and $\alpha$-amylase inhibitory [7], antimicrobial, immunostimulatory [7], angiogenesis inhibitory [8], tyrosinase inhibitory activities [9], promotion of melanin biosynthesis [9], and prevention of endothelial 
senescence [10]. Recently, Tatefuji et al. also reported that acute and subchronic MSE administration showed no adverse effect in rat [11]. In human study, MSE administration decreases the serum uric acid levels by inhibiting the reabsorption of uric acid in the renal tubular epithelia as well as by increasing the HDL cholesterol levels by PPAR agonistic activity with no cause of the damage to health [12]. Furthermore, Tani et al. reported that single and repeated administration of MSE demonstrated no clinical noteworthy abnormalities [13]. MSE contains $1.2 \mathrm{mg} / \mathrm{g}(5.26 \mu \mathrm{mol} / \mathrm{g})$ of RSV [13], while average content of RSV was $1.04 \mathrm{mg} / \mathrm{L}$ in red wine [14]. Therefore, MSE becomes a nutrient source of RSV with harmless long-term intake. RSV has been identified as a Sirt1 activator that has been shown to protect various organs against aging $[15,16]$. Furthermore, RSV possesses antioxidative activity and protective effect of ROS- and ultraviolet-induced cell death $[17,18]$. In addition, Sirt1 is a key modulator of cellular pathways involved in inherited dermatologic diseases and skin cancers [19], suggesting that Sirt1 activation is a molecular target for dermatological therapy. In the present study, we investigated antiatrophic effects of MSE and RSV on agerelated skin pathologies in $\mathrm{Sod1}^{-/-}$mice.

\section{Materials and Methods}

2.1. Reagents. MSE (Lot number YMP-M-110115) was provided by the Institute for Bee Products \& Health Science, Yamada Bee Company, Inc. (Okayama, Japan). The MSE contains trans-resveratrol (RSV, $0.10 \% \mathrm{w} / \mathrm{w}$ ), gnetin C $(2.03 \% \mathrm{w} / \mathrm{w})$, gnemonoside A $(16.35 \% \mathrm{w} / \mathrm{w})$, and gnemonoside D $(3.97 \% \mathrm{w} / \mathrm{w})$. Resveratrol (RSV) was obtained from Tokyo Chemical Industry Co. Ltd. (CAS 501-36-0, Tokyo, Japan). The purity of RSV is more than $98 \%$.

2.2. Mice and Diets. Sod1 ${ }^{-/-}$mice were purchased from the Jackson Laboratory (Bar Hrbor, ME, USA). The genotyping of Sod1 $1^{-/-}$allele was performed using genomic PCR with genomic DNA isolated from the tail tip, as previously reported [20]. The animals were housed in a room temperature of $24 \pm 1^{\circ} \mathrm{C}$, a relative humidity of $55 \pm 10 \%$, and a $12 \mathrm{~h}$ light/dark cycle and were fed ad libitum. Experimental procedures were approved by the Animal Care and Use Committee of Chiba University. At 4 weeks of age, mice were randomly divided into four groups and fed respective experimental diets for 12 weeks: control MF diet (composition: 7.9\% water, $21.1 \%$ protein, $5.1 \%$ lipid, $5.8 \%$ ash, $2.8 \%$ fiber, and $55.3 \%$ soluble nitrogen free extract, $359 \mathrm{kcal} / 100 \mathrm{~g}$, Oriental Yeast Co., Ltd., Tokyo, Japan), control MF diet containing 0.04\% (w/w) RSV as previously described [15], and control MF diets containing $0.1 \%$ or $0.5 \%(\mathrm{w} / \mathrm{w})$ MSE (Lot number YMP-M110115) according to the previous study [8].

2.3. Histology. For histological morphology, skin specimens from back tissues were dissected and fixed in a $20 \%$ formalin neutral buffer solution (Wako, Osaka, Japan) overnight. After dehydration and penetration, skin specimens were embedded in paraffin and sectioned on a microtome (ROM-380, Yamato Koki Kogyo Co. Ltd., Saitama, Japan) at $4 \mu \mathrm{m}$ thickness by standard techniques. Hematoxylin and eosin staining for skin morphology and Sirius red staining for total collagen deposition were performed as described previously [21-23]. The thickness of the skin tissue was measured using Leica QWin V3 image software (Leica, Germany).

2.4. Measurement of Oxidative Stress Markers. In order to measure the 8-isoprostane content, blood was collected from the left ventricular space and centrifuged at 12,000 rpm for $5 \mathrm{~min}$ at room temperature. Plasma was separated from the clotted blood and added $100 \mu \mathrm{M}$ indomethacin and $0.005 \%$ dibutylhydroxytoluene. The 8 -isoprostane level was measured using the 8-isoprostane EIA Kit (Cayman Chemical Company) according to the manufacturer's instructions. The plasma was also assayed for the protein concentration using the DC Protein Assay Kit (Bio-Rad, Hercules, CA, USA), and 8 -isoprostane levels were normalized to the protein level.

For intracellular ROS measurement, bone marrow cells $\left(5-10 \times 10^{5}\right.$ cells/two tibias of a mouse) were collected by flushing tibias with phosphate-buffered saline using 26-G needles and stained with $10 \mu \mathrm{M}$ of CM- $\mathrm{H}_{2}$ DCFDA (DCF, Life Technologies Corporation) for $30 \mathrm{~min}$ at $37^{\circ} \mathrm{C}$. Primary dermal fibroblasts were incubated with $10 \mu \mathrm{M}$ DCF for $30 \mathrm{~min}$ at $37^{\circ} \mathrm{C}$. After incubation, cells were trypsinized and resuspended in PBS. Their fluorescence intensities were assessed using a flow cytometer (BD FACSCanto II, BD Biosciences).

2.5. Cell Culture. Skin tissues were dissected from Sod1 $1^{-1-}$ neonates at 5 days of age. The primary dermal fibroblasts were isolated by dissociation in $0.2 \%$ collagenase type 2 (Worthington Biochemical Corporation Lakewood, NJ, USA) at $37^{\circ} \mathrm{C}$ for $60 \mathrm{~min}$. Cells were cultured in $\alpha$-MEM (Life Technologies Corporation, Carlsbad, CA, USA) supplemented with $20 \%$ fetal bovine serum (FBS), 100 unit/mL penicillin, and $0.1 \mathrm{mg} / \mathrm{mL}$ streptomycin at $37^{\circ} \mathrm{C}$ in a humidified incubator with $5 \% \mathrm{CO}_{2}$ and $1 \% \mathrm{O}_{2}$. Cells were treated with $10 \mu \mathrm{M}$ RSV at $72 \mathrm{~h}$. We determined the concentration and duration of RSV treatment in this study according to our previous paper [6].

2.6. Outgrowth Assay. The back skin was sterilized with $70 \%$ ethanol, rinsed with PBS (Takara Bio Inc., Shiga, Japan), and punched out into discs measuring $5 \mathrm{~mm}$ in diameter using dermal punch (Nipro, Tokyo, Japan). The punched skin discs were placed into a 12 -well culture plate (Falcon BD, Franklin Lakes, NJ) and cultured with or without $10 \mu \mathrm{M}$ RSV in $\alpha$ MEM containing 20\% FBS, 100 units/mL of penicillin, and $0.1 \mathrm{mg} / \mathrm{mL}$ of streptomycin at $37^{\circ} \mathrm{C}$ in a humidified incubator with $5 \% \mathrm{CO}_{2}$ and $1 \% \mathrm{O}_{2}$. The number of outgrowth fibroblasts originating from the mouse skin disc was directly counted at $72 \mathrm{~h}$ after culture. The method of this experiment was performed as described previously [5].

2.7. Quantitative PCR. Total RNA was extracted from back skin using the Trizol reagent (Life Technologies Corporation, Carlsbad, CA, USA) according to the manufacturer's instructions. cDNA was synthesized from $1 \mu \mathrm{g}$ of total RNA using reverse transcriptase (ReverTra Ace qPCR RT Master Mix, Toyobo, Osaka, Japan). Real-time PCR was performed on 
a MiniOpticon (Bio-Rad) with the SYBR Green PCR Master Mix (Bio-Rad) according to the manufacturer's instructions. All data were normalized to the level of the housekeeping gene glyceraldehyde-3-phosphate dehydrogenase (Gapdh). The following primers were used for the analysis: Gapdh, forward, 5' -ATGTGTCCGTCGTGGATCTGA-3', and reverse, $5^{\prime}$-TGCCTGCTTCACCACCTTCT-3'; Collal, forward, $5^{\prime}$ CATGTTCAGCTTTGTGGACCT- $3^{\prime}$, and reverse, $5^{\prime}$-GCAGCTGACTTCAGGGATGT-3' ; p53, forward, $5^{\prime}$-ACGCTTCTCCGAAGACTGG-3' , and reverse, $5^{\prime}$-AGGGAGCTCGAGGCTGATA-3'; Sirt1, forward, $5^{\prime}$-CAGTGAGAAAATGCTGGCCTA-3', and reverse, $5^{\prime}$-TTGGTGGTACAAACAGGTATTGA-3'.

2.8. Statics. The statistical analyses were performed using Student's $t$-test for comparisons between two groups and Tukey's test for comparisons among three groups. Differences between the data were considered significant when the $P$ values were less than 0.05 . All data are expressed as the mean \pm standard deviation (SD).

\section{Results}

3.1. MSE and RSV Attenuate the Skin Atrophy of Sod1 ${ }^{-/-}$Mice. SOD1, one of the cellular antioxidant enzymes, plays a pivotal role in regulating oxidative and reductive balance. Sod1 ${ }^{-/-}$ mice showed age-related atrophic morphology in their skin accompanied by the degeneration of collagen [5]. Therefore, we have used Sod1 ${ }^{-1-}$ mice for skin aging research and for screening of antiatrophic compounds in skin thickness [2426]. In this context, we investigated antiatrophic effects of MSE and RSV on the skin thickness of Sod1 $1^{-/-}$mice.

In a preliminary experiment, MF diets containing 5\% or $0.5 \%$ MSE were orally administrated to the Sod1 $1^{+/+}$and Sod1 $^{-/-}$mice daily for 12 weeks beginning at 4 weeks of age. The results showed that both MSE diets improved the skin thickness of Sod1 ${ }^{-/-}$mice and there were no adverse effects of skin pathologies of Sod1 ${ }^{+/+}$mice (data not shown). Therefore, we selected the control diet containing 0.5\% MSE to confirm antiatrophic effect on Sod1 ${ }^{-/-}$skin. MSE and RSV were orally administered to the $S o d 1^{+/+}$and $S o d 1^{-/-}$mice under the same conditions. As shown in Figure 1(a), the skin of Sod1 $1^{-/-}$ mice was significantly thinner compared to that of Sod1 $1^{+/+}$ mice, confirming skin atrophy in Sod1 ${ }^{-/-}$mice. The back skin of Sod1 ${ }^{-/-}$that had been administrated with the MSE diets was significantly thicker compared to that of Sod1 $1^{-/-}$ mice treated with the control diet (Figures $1(\mathrm{~b})-1(\mathrm{~d})$ ). RSV diet also improved skin atrophy of Sod1 ${ }^{-1-}$ mice compared to Sod1 $^{-/-}$mice treated with the control diet (Figures 1(b)-1(d)). To investigate the adverse effect of MSE and RSV diets, we similarly administered MSE and RSV to the Sod $1^{+/+}$mice. No significant difference in skin thickness and morphology was observed in Sod1 ${ }^{+/+}$mice treated with MSE and RSV (data not shown), indicating that MSE and RSV were safety food factors in skin during short-time treatment. In addition, Sirius red staining revealed that the skin of Sod1 ${ }^{-/-}$mice was decreased in staining intensity compared to that observed in Sod1 $1^{+/+}$ mice (Figure 2(a)), confirming dermal collagen decline in Sod $1^{-/-}$mice. Notably, both MSE and RSV diets increased the Sirius red intensity in Sod1 ${ }^{-/-}$dermis (Figure 2(a)), implying enhancement of collagen level in Sod1 ${ }^{-/}$skin.

3.2. MSE and RSV Alter Gene Expression in Sod1 ${ }^{-1-}$ Skin. To investigate skin atrophy-preventing mechanism of MSE and RSV on skin atrophy in Sod1 ${ }^{-/-}$, we analyzed expression patterns of type I collagen and age-related genes in skin. In Sod1 $^{-/-}$skin, mRNA level of Colla1 was significantly downregulated compared to those of $\mathrm{SodI}^{+/+}$, indicating reduced collagen biosynthesis (Figure 2(b)). Moreover, p53, one of the major age-related genes, also significantly upregulated in Sod1 $1^{-1-}$ skin (Figure 2(c)). MSE and RSV treatment significantly normalized mRNA levels of Colla1 and p53 in Sod1 ${ }^{-/-}$ skin (Figures 2(b) and 2(c)). Interestingly, we revealed that MSE and RSV treatment also significantly upregulated Sirt1 expression, suggesting the molecular link between Sirt1 expression and skin thinning in Sod1 ${ }^{-/-}$mice (Figure 2(d)). These findings demonstrated that application of MSE and RSV diets improved the skin atrophy accompanied by normalization and activation of age-related genes in Sod1 ${ }^{-/-}$ mice.

\subsection{MSE and RSV Significantly Attenuate Oxidative Damage} in Sod1 ${ }^{-1-}$ Mice. Sod1 ${ }^{-1-}$ mice showed significant increase of several oxidative damage markers, including lipid peroxidation, in tissues $[20,24,27,28]$. In order to evaluate oxidative damage, we measured the lipid peroxidation levels in the plasma. Regarding the 8-isoprostane levels, MSE and RSV containing diets significantly reduced the 8-isoprostane content in the plasma (Figure 3(a)). Furthermore, MSE and RSV containing diets decreased intracellular ROS level in cells from bone marrow (Figure 3(b)). These data indicate that MSE and RSV treatment mitigated oxidative damage in Sod1 ${ }^{-/-}$mice.

3.4. MSE and RSV Significantly Restore Viability in Sod1 ${ }^{-/-}$ Fibroblasts. We investigated whether the RSV treatment attenuated intracellular ROS production and promoted the proliferation of Sod1 $1^{-/-}$fibroblasts in vitro. Preliminary experiments revealed that RSV treatment for $24 \mathrm{~h}$ with various concentrations of 30 to $100 \mu \mathrm{M}$ slightly suppressed cell viability of Sod1 $1^{+/+}$fibroblasts. In contrast, $10 \mu \mathrm{M}$ RSV treatment for $72 \mathrm{~h}$ showed no adverse effect of cell viability in Sod $^{+/+}$fibroblasts. Therefore, we determined dose and duration of the RSV experiment in vitro. Flow cytometer analysis indicated that RSV treatment significantly decreased intracellular ROS generation in Sod1 ${ }^{-/-}$fibroblasts (Figure 3(c)). Moreover, the organ culture experiments using skin discs revealed that the Sod1 ${ }^{-/-}$fibroblasts showed marked suppression of their outgrowth capacity compared to that observed in the Sod1 $1^{+/+}$mice (Figure 4(a)). Treatment with $10 \mu \mathrm{M}$ RSV significantly enhanced the fibroblasts outgrowth activity 


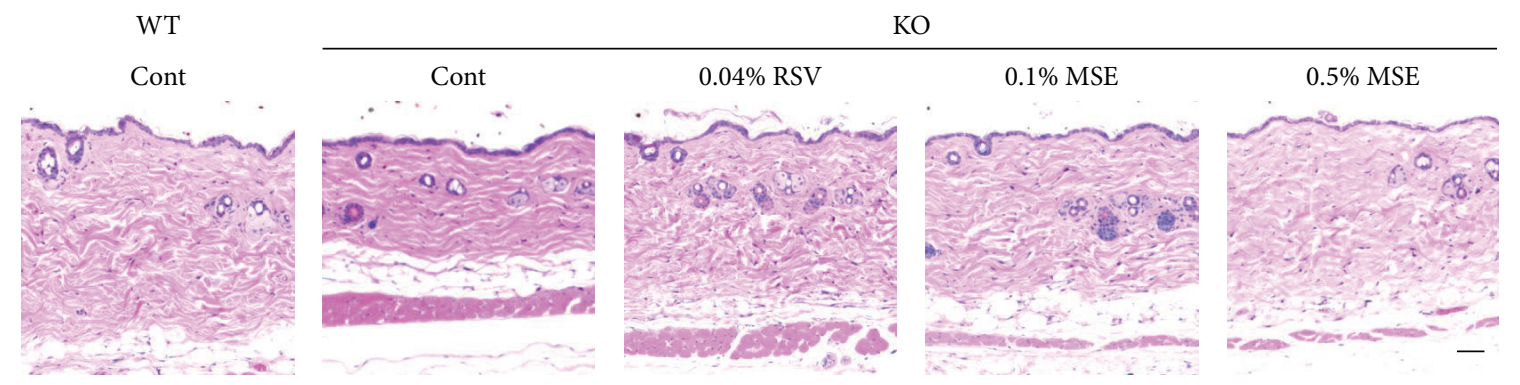

(a)

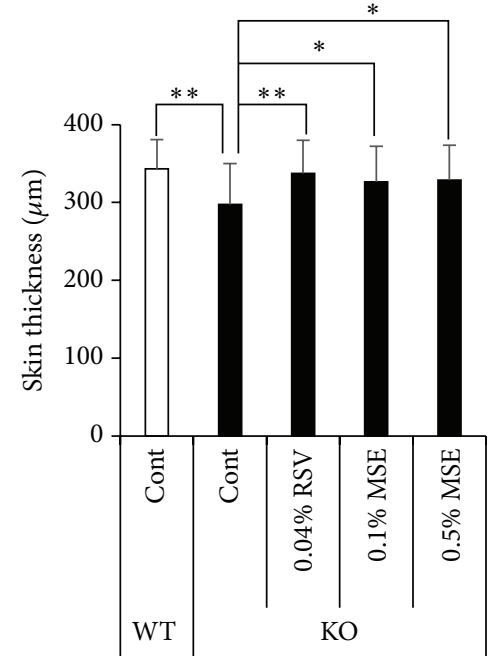

(b)

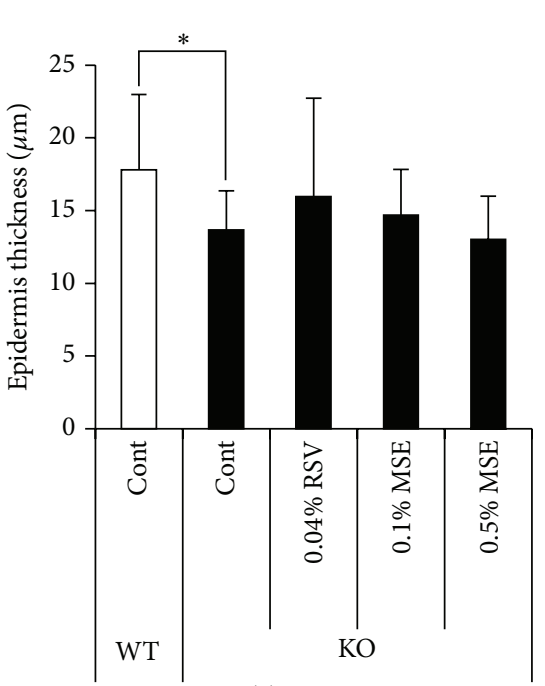

(c)

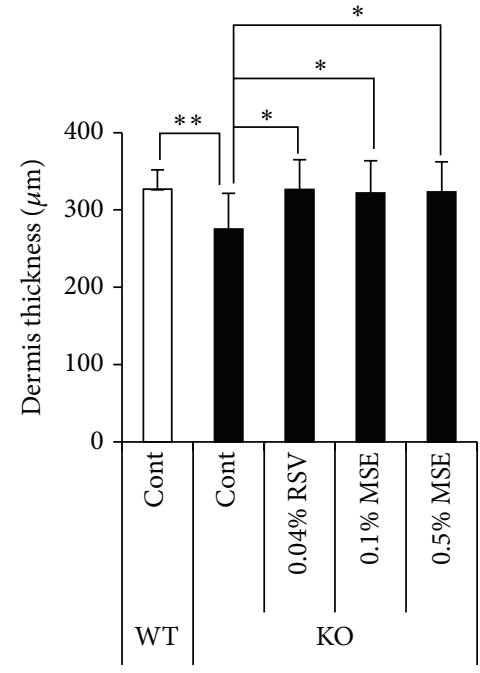

(d)

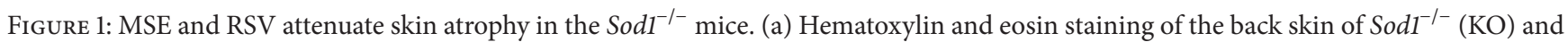
Sodl $^{+/+}$(WT) mice treated with the MSE or RSV. MSE and RSV containing diets were administrated for 12 weeks. The thickness of (b) total, (c) epidermis, and (d) dermis of the back skin of the Sod1 $1^{-/-}$and Sod1 $1^{+/+}$mice treated with MSE or RSV $(n=10-12)$. The statistical evaluations were performed using the Tukey's test. These data indicate the mean $\pm \mathrm{SD} ;{ }^{*} P<0.05,{ }^{* *} P<0.01$. The scale bar represents $100 \mu \mathrm{m}$.

from the Sod1 ${ }^{-/}$skin discs (Figure 4(b)). These findings collectively suggested that the RSV promoted the migration and proliferation of Sodl ${ }^{-1-}$ fibroblasts in vitro.

\section{Discussion}

In the present study, we demonstrated that MSE and RSV significantly reversed skin thinning via reduction of oxidative damages in Sod1 ${ }^{-1-}$ mice (Figure 1). Recently, we have reported that Sod1 ${ }^{-/-}$fibroblasts showed excessive ROS accumulation associated with mitochondrial dysfunction [6]. In vitro study also revealed that RSV treatment significantly reduced intracellular ROS generation and restored cell viability in Sod1 $1^{-1-}$ fibroblasts (Figures 3(c) and 4). Accumulating evidence revealed that RSV activates mitochondrial function and antioxidant defense leading to suppressing ROS generation [29]. Furthermore, SIRT1 also increases mitochondrial function and biogenesis and promotes cell proliferation and migration $[15,30,31]$. In a human study, treatment with a nutraceutical supplement containing resveratrol, procyanidin, and ellagic acid induced reduction of skin wrinkling, as well as reducing systemic and skin oxidative stress in a clinical setting [32]. These findings suggested that the Sirt1-mediated antioxidant activities of RSV contribute to attenuate skin damages in mammals. To rescue agerelated changes in tissues of Sod1 ${ }^{-/-}$mice, we have evaluated beneficial effects of several antioxidants in vivo. Ascorbic acid administration significantly attenuated bone loss and fragility of Sod1 ${ }^{-1-}$ mice [28]. Transdermal administration of ascorbic acid derivatives also normalized skin thinning of Sodl $1^{-/-}$ mice $[25,26]$. Furthermore, Iuchi et al. reported that oral $\mathrm{N}$-acetylcysteine treatment mitigated hemolytic anemia of Sod1 ${ }^{-1-}$ mice by suppressed ROS generation in red blood cells [27]. Recently, Shibuya et al. showed that an SOD/catalase mimetic, PAPLAL, treatment attenuated skin atrophy [33]. These reports strongly supported that antioxidants, such as RSV, ascorbic acid, N-acetylcysteine, and PAPLAL, positively improved oxidative damage-induced organ pathologies.

As shown in Figure 2(c), Sod1 deficiency showed upregulation of $p 53$ gene expression, which regulates cellular senescence and death, in skin (Figure 2(c)). We previously reported that Sod1 loss induced $\mathrm{O}_{2}{ }^{-}$generation and upregulated p53 protein level in skin fibroblasts [6]. Ascorbic acid derivatives significantly downregulated p53 expression and improved 

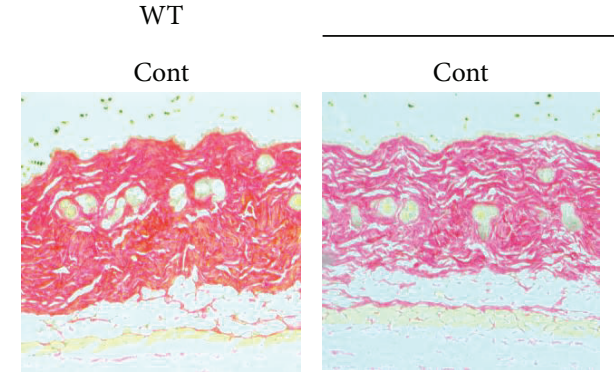

$\mathrm{KO}$

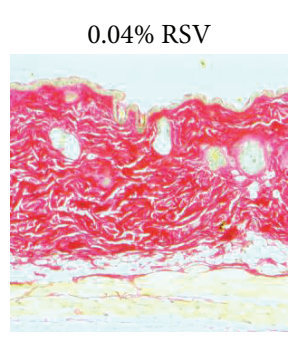

(a)

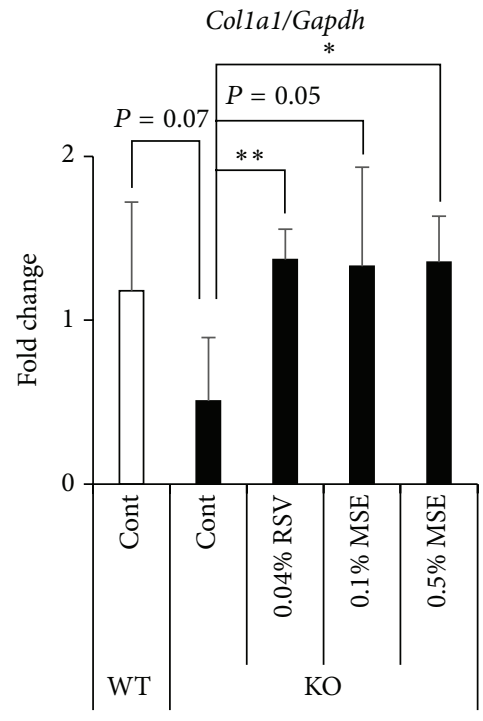

(b)

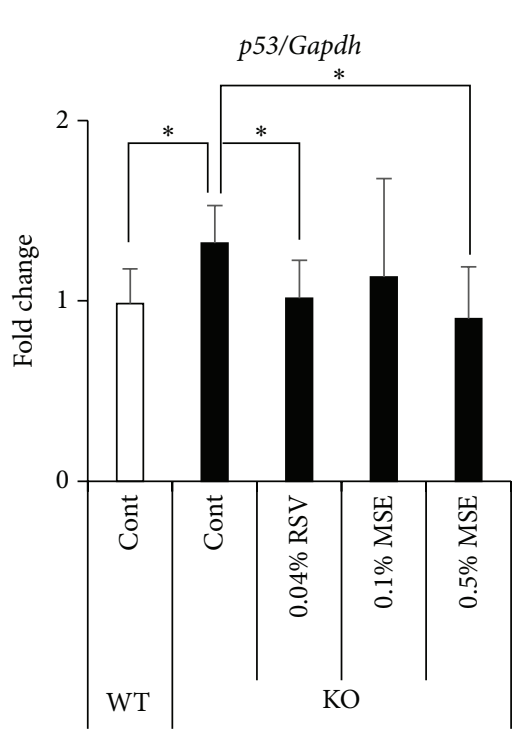

(c)

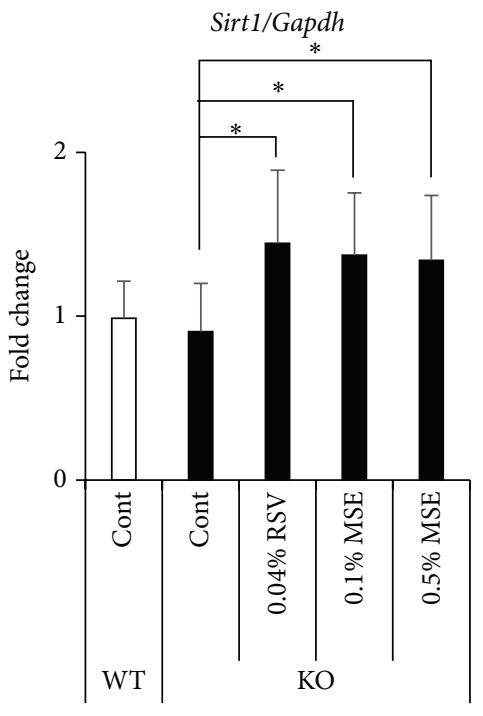

(d)

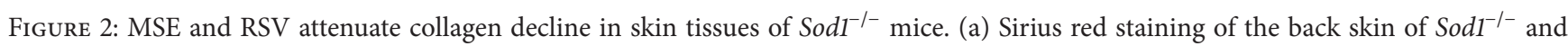
$\mathrm{Sodl}^{+/+}$mice treated with the MSE or RSV. Relative mRNA expression of (b) Sirt1, (c) Colla1, and (d) p53. Each of the mRNA expressions was determined by qRT-PCR $(n=8-12)$. The statistical evaluations were performed using the two-tailed Student's $t$-test for unpaired values. These data indicate the mean $\pm \mathrm{SD} ;{ }^{*} P<0.05,{ }^{* *} P<0.01$. The scale bar represents $100 \mu \mathrm{m}$.

cell viability in Sod1 ${ }^{-1-}$ fibroblasts $[6,25]$. In a genetically modified model, p53 activation induced accelerated aginglike phenotypes, including skin atrophy, in p53 mutant mice [34]. Gannon et al. also reported that p53 activation by $M d m 2$-specific loss in keratinocytes induced epidermal stem cell senescence and atrophy in mice, suggesting that p53 activation in skin accelerated aging-like skin thinning in mice [35]. MSE and RSV treatment significantly downregulated mRNA level of $p 53$ in Sod1 $1^{-1-}$ skin (Figure 2(c)). These data indicated that MSE and RSV treatment may delay skin aging via reducing the p53 upregulation in skin.

RSV promotes the activity and expression of Sirt1 [15]. MSE and RSV also normalized the gene expression of Colla1 and upregulated the gene expression of Sirt1 in skin of Sod1 $1^{-/-}$ mice (Figures 2(b) and 2(d)). Sirt1 upregulation by RSV may protect skin aging from oxidative damage in $\mathrm{Sod1}^{-1-}$ mice. Actually, Lee et al. reported that RSV treatment or Sirt1 overexpression significantly inhibited matrix metalloprotease- 9 expression and appeared to protect collagen from degradation after ultraviolet radiation in human dermal fibroblasts and skin tissues [36]. Serravallo et al. reported that Sirt1 plays a pivotal role in modulating skin diseases including psoriasis, autoimmune disease, cutaneous fungal infection, inherited dermatological diseases, and cancer [19]. These findings indicated that upregulation of Sirtl expression protected skin damages in vivo.

Recently, Konno et al. reported that RSV and MSE showed the agonistic activity for PPAR $\alpha$ and PPAR $\gamma$ in vitro [12]. It is reported that a PPAR $\alpha / \gamma$ dual agonist, MHY966, treatment significantly suppressed UVB-induced collagen digestion, lipid peroxidation, and inflammatory response via activating PPAR $\alpha$ and PPAR $\gamma$ in mouse skin during photoaging [37]. Moreover, Mastrofrancesco et al. reported that PPAR $\gamma$ activation in skin normalized inflammatory response in IL-21-induced epithelial hyperplasia in mice [38]. These reports suggested that RSV and MSE may activate PPAR $\alpha$ and PPAR $\gamma$ leading to attenuating the skin atrophy in Sodr ${ }^{-/-}$ mice.

Finally, we, here, focused on RSV in MSE and antiatrophic effects of RSV in Sodl ${ }^{-/-}$skin. Since MSE also contains several RSV-derivatives such as gnetin C, gnemonoside A, and gnemonoside $\mathrm{D}$, we cannot rule out antiatrophic effects of 


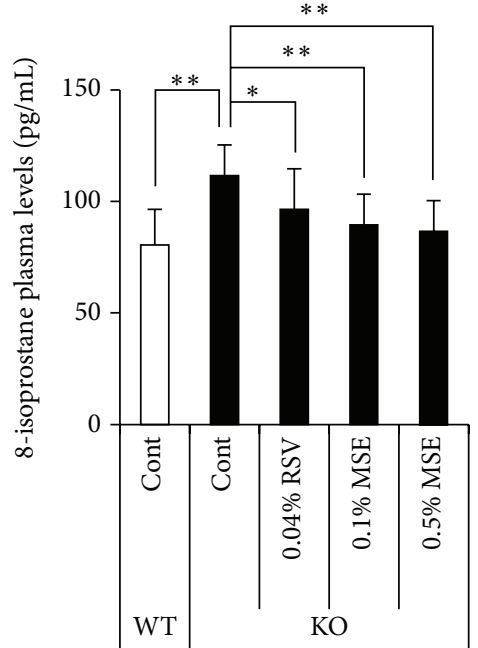

(a)

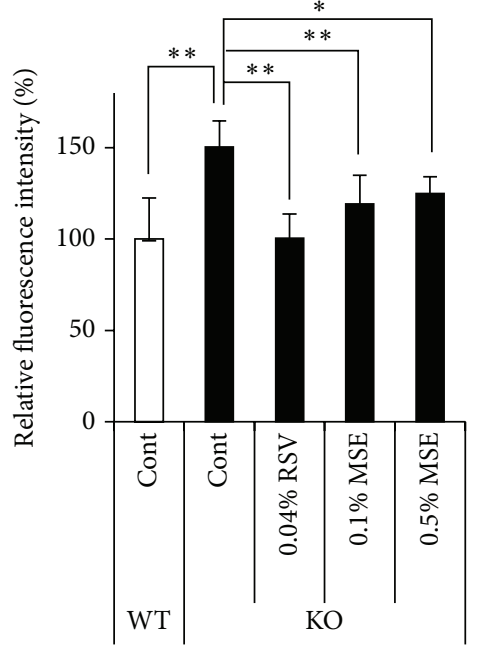

(b)

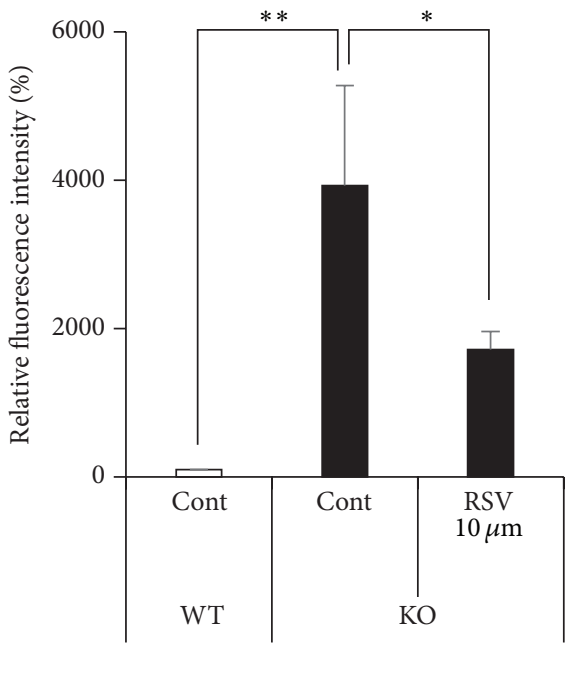

(c)

FIGURE 3: MSE and RSV decrease oxidative damage and ROS production. (a) 8-isoprostane content in plasma obtained from Sod1 $1^{-/-}$and $S_{\text {Sodl }}{ }^{+/}$mice treated with MSE and RSV $(n=10-12)$. (b) The intracellular ROS levels of bone marrow cells of Sod1 $1^{-/-}$and Sod1 $1^{+/+}$mice were measured using a DCF dye $(n=5-6)$. (c) The relative intracellular ROS level in Sodr $1^{-/}$fibroblasts treated with $10 \mu \mathrm{M}$ RSV for $72 \mathrm{~h}$ was measured by a DCF dye $(n=3)$. The statistical evaluations were performed using the Tukey's test. These data indicate the mean \pm SD; ${ }^{*} P<0.05,{ }^{* *} P<0.01$.
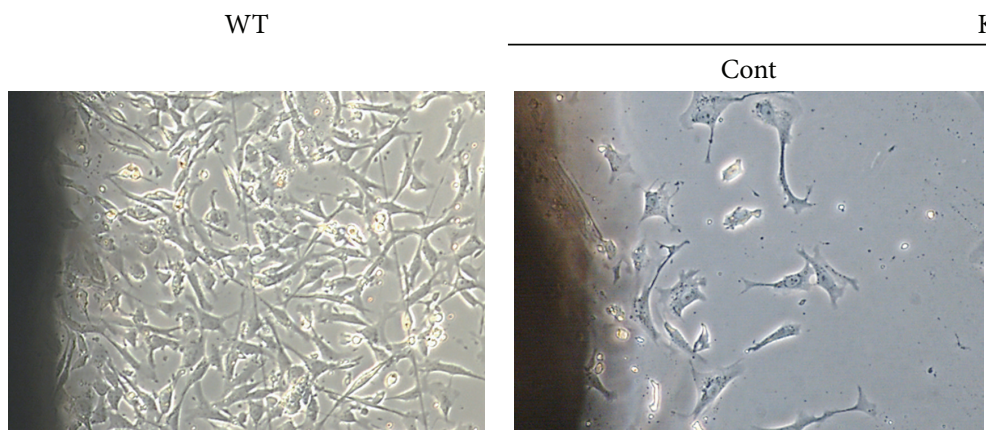

(a)

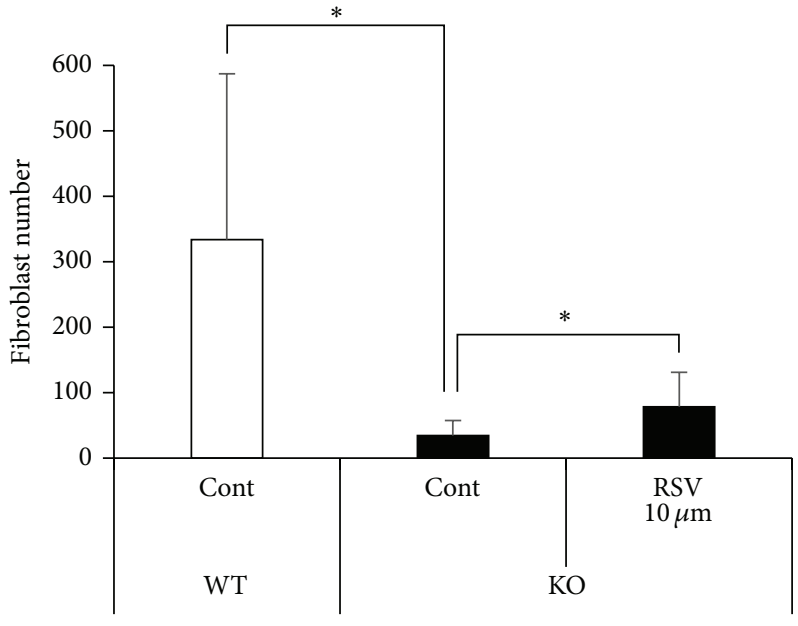

(b)

$\mathrm{KO}$

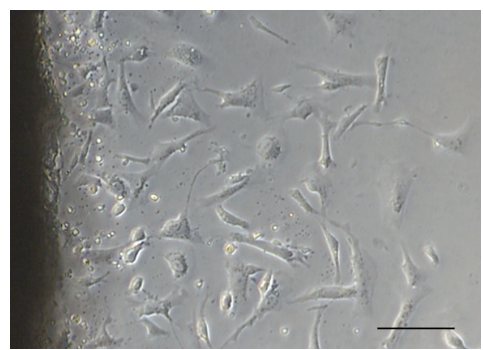

RSV 
the RSV derivatives in MSE. Further analysis should be needed to clarify the beneficial effect of other RSV derivatives in MSE on skin atrophy in Sod1 $1^{-1-}$ mice.

\section{Conclusion}

In the present study, we demonstrated that MSE and RSV treatment effectively attenuated aging-like skin pathologies accompanied by upregulation of Sirt1 expression in Sod1 $1^{-/-}$ skin. MSE and RSV also exhibited less adverse effect on skin morphology. Consistent with our results, many interventions reported safety of MSE and RSV treatment in human. Therefore, MSE is useful for nutrient source of RSV as well as safety antioxidant for delaying skin aging in humans.

\section{Conflict of Interests}

This research was supported by the institute for Bee Products $\&$ Health Science, Yamada Bee Company, Inc.

\section{Acknowledgments}

The authors thank Tomoki Ikuta, Keiko Kobayashi, and Tomoki Tatefuji (Yamada Bee Company) for providing MSE and helpful discussions. They also thank Dr. Keiji Kobayashi, Dr. Masato Koike, and Toshihiko Toda, from Chiba University, for their valuable technical assistance.

\section{References}

[1] B. Poljsak, R. G. Dahmane, and A. Godic, "Intrinsic skin aging: the role of oxidative stress," Acta Dermatovenerol Alp Panonica Adriat, vol. 21, no. 2, pp. 33-36, 2012.

[2] S. Shuster, M. M. Black, and E. McVitie, "The influence of age and sex on skin thickness, skin collagen and density," British Journal of Dermatology, vol. 93, no. 6, pp. 639-643, 1975.

[3] T. Finkel and N. J. Holbrook, "Oxidants, oxidative stress and the biology of ageing," Nature, vol. 408, no. 6809, pp. 239-247, 2000.

[4] T. Shimizu, H. Nojiri, S. Kawakami, S. Uchiyama, and T. Shirasawa, "Model mice for tissue-specific deletion of the manganese superoxide dismutase gene," Geriatrics \& Gerontology International, vol. 10, supplement 1, pp. S70-S79, 2010.

[5] S. Shibuya, Y. Ozawa, T. Toda et al., "Collagen peptide and vitamin C additively attenuate age-related skin atrophy in Sod1deficient mice," Bioscience, Biotechnology, and Biochemistry, vol. 78, no. 7, pp. 1212-1220, 2014.

[6] K. Watanabe, S. Shibuya, H. Koyama et al., "Sod1 loss induces intrinsic superoxide accumulation leading to p53-mediated growth arrest and apoptosis," International Journal of Molecular Sciences, vol. 14, no. 6, pp. 10998-11010, 2013.

[7] E. Kato, Y. Tokunaga, and F. Sakan, "Stilbenoids isolated from the seeds of melinjo (Gnetum gnemon L.) and their biological activity," Journal of Agricultural and Food Chemistry, vol. 57, no. 6, pp. 2544-2549, 2009.

[8] K. Kunimasa, T. Ohta, H. Tani et al., "Resveratrol derivativerich melinjo (Gnetum gnemon L.) seed extract suppresses multiple angiogenesis-related endothelial cell functions and tumor angiogenesis," Molecular Nutrition and Food Research, vol. 55, no. 11, pp. 1730-1734, 2011.
[9] M. Yanagihara, M. Yoshimatsu, A. Inoue, T. Kanno, T. Tatefuji, and K. Hashimoto, "Inhibitory effect of gnetin C, a resveratrol dimer from melinjo (Gnetum gnemon), on tyrosinase activity and melanin biosynthesis," Biological and Pharmaceutical Bulletin, vol. 35, no. 6, pp. 993-996, 2012.

[10] H. Ota, M. Akishita, H. Tani et al., "trans-resveratrol in Gnetum gnemon protects against oxidative-stress-induced endothelial senescence," Journal of Natural Products, vol. 76, no. 7, pp. 1242 1247, 2013.

[11] T. Tatefuji, M. Yanagihara, S. Fukushima, and K. Hashimoto, "Safety assessment of melinjo (Gnetum gnemon L.) seed extract: acute and subchronic toxicity studies," Food and Chemical Toxicology, vol. 67, pp. 230-235, 2014.

[12] H. Konno, Y. Kanai, M. Katagiri et al., "Melinjo (Gnetum gnemon L.) seed extract decreases serum uric acid levels in nonobese Japanese males: a randomized controlled study," Evidence-Based Complementary and Alternative Medicine, vol. 2013, Article ID 589169, 9 pages, 2013.

[13] H. Tani, S. Hikami, S. Iizuna et al., "Pharmacokinetics and safety of resveratrol derivatives in humans after oral administration of melinjo (Gnetum gnemon L.) seed extract powder," Journal of Agricultural and Food Chemistry, vol. 62, no. 8, pp. 1999-2007, 2014.

[14] M. Sato, Y. Suzuki, T. Okuda, and K. Yokotsuka, "Contents of resveratrol, piceid, and their isomers in commercially available wines made from grapes cultivated in Japan," Bioscience, Biotechnology and Biochemistry, vol. 61, no. 11, pp. 1800-1805, 1997.

[15] J. A. Baur and D. A. Sinclair, "Therapeutic potential of resveratrol: the in vivo evidence," Nature Reviews Drug Discovery, vol. 5, no. 6, pp. 493-506, 2006.

[16] B. P. Hubbard and D. A. Sinclair, "Small molecule SIRT1 activators for the treatment of aging and age-related diseases," Trends in Pharmacological Sciences, vol. 35, no. 3, pp. 146-154, 2014.

[17] J.-H. Jang and Y.-J. Surh, "Protective effects of resveratrol on hydrogen peroxide-induced apoptosis in rat pheochromocytoma (PC12) cells," Mutation Research: Genetic Toxicology and Environmental Mutagenesis, vol. 496, no. 1-2, pp. 181-190, 2001.

[18] V. M. Adhami, F. Afaq, and N. Ahmad, "Suppression of ultraviolet B exposure-mediated activation of NF- $\kappa$ B in normal human keratinocytes by resveratrol," Neoplasia, vol. 5, no. 1, pp. 74-82, 2003.

[19] M. Serravallo, J. Jagdeo, S. A. Glick, D. M. Siegel, and N. I. Brody, "Sirtuins in dermatology: applications for future research and therapeutics," Archives of Dermatological Research, vol. 305, no. 4, pp. 269-282, 2013.

[20] D. Morikawa, H. Nojiri, Y. Saita et al., "Cytoplasmic reactive oxygen species and SOD1 regulate bone mass during mechanical unloading," Journal of Bone and Mineral Research, vol. 28, no. 11, pp. 2368-2380, 2013.

[21] S. Uchiyama, T. Shimizu, and T. Shirasawa, "CuZn-SOD deficiency causes ApoB degradation and induces hepatic lipid accumulation by impaired lipoprotein secretion in mice," The Journal of Biological Chemistry, vol. 281, no. 42, pp. 31713-31719, 2006.

[22] H. Nojiri, T. Shimizu, M. Funakoshi et al., "Oxidative stress causes heart failure with impaired mitochondrial respiration," Journal of Biological Chemistry, vol. 281, no. 44, pp. 33789-33801, 2006.

[23] A. C. Nauta, M. Grova, D. T. Montoro et al., "Evidence that mast cells are not required for healing of splinted cutaneous 
excisional wounds in mice," PLOS ONE, vol. 8, no. 3, Article ID e59167, 2013.

[24] K. Murakami, J. Inagaki, M. Saito et al., "Skin atrophy in cytoplasmic SOD-deficient mice and its complete recovery using a vitamin C derivative," Biochemical and Biophysical Research Communications, vol. 382, no. 2, pp. 457-461, 2009.

[25] S. Shibuya, K. Kinoshita, and T. Shimizu, "Protective effects of vitamin $\mathrm{C}$ derivatives on skin atrophy caused by Sodl deficiency," in Handbook of Diet, Nutrition and the Skin, V. R. Preedy, Ed., pp. 351-364, Wageningen Academic, 2012.

[26] S. Shibuya, H. Nojiri, D. Morikawa, H. Koyama, and T. Shimizu, "Protective effects of vitamin $\mathrm{C}$ on age-related bone and skin phenotypes caused by intracellular reactive oxygen species," in Oxidative Stress and Dietary Antioxidants, V. R. Preedy, Ed., pp. 137-155, Academic Press, 2013.

[27] Y. Iuchi, F. Okada, K. Onuma et al., "Elevated oxidative stress in erythrocytes due to a SOD1 deficiency causes anaemia and triggers autoantibody production," Biochemical Journal, vol. 402, no. 2, pp. 219-227, 2007.

[28] H. Nojiri, Y. Saita, D. Morikawa et al., "Cytoplasmic superoxide causes bone fragility owing to low-turnover osteoporosis and impaired collagen cross-linking," Journal of Bone and Mineral Research, vol. 26, no. 11, pp. 2682-2694, 2011.

[29] Z. Ungvari, W. E. Sonntag, R. de Cabo, J. A. Baur, and A. Csiszar, "Mitochondrial protection by resveratrol," Exercise and Sport Sciences Reviews, vol. 39, no. 3, pp. 128-132, 2011.

[30] Y. Zhang, M. Zhang, H. Dong et al., "Deacetylation of cortactin by SIRT1 promotes cell migration," Oncogene, vol. 28, no. 3, pp. 445-460, 2009.

[31] N. L. Price, A. P. Gomes, A. J. Y. Ling et al., "SIRT1 is required for AMPK activation and the beneficial effects of resveratrol on mitochondrial function," Cell Metabolism, vol. 15, no. 5, pp. 675690, 2012.

[32] D. Buonocore, A. Lazzeretti, P. Tocabens et al., "Resveratrolprocyanidin blend: nutraceutical and antiaging efficacy evaluated in a placebo-controlled, double-blind study," Clinical, Cosmetic and Investigational Dermatology, vol. 5, pp. 159-165, 2012.

[33] S. Shibuya, Y. Ozawa, K. Watanabe et al., "Palladium and platinum nanoparticles attenuate aging-like skin atrophy via antioxidant activity in mice," PLoS ONE, vol. 9, no. 10, Article ID e109288, 2014.

[34] S. D. Tyner, S. Venkatachalam, J. Choi et al., "p53 mutant mice that display early ageing-associated phenotypes," Nature, vol. 415, no. 6867, pp. 45-53, 2002.

[35] H. S. Gannon, L. A. Donehower, S. Lyle, and S. N. Jones, "Mdm2-p53 signaling regulates epidermal stem cell senescence and premature aging phenotypes in mouse skin," Developmental Biology, vol. 353, no. 1, pp. 1-9, 2011.

[36] J. S. Lee, K. Y. Park, H. G. Min et al., "Negative regulation of stress-induced matrix metalloproteinase- 9 by Sirtl in skin tissue," Experimental Dermatology, vol.19, no. 12, pp. 1060-1066, 2010.

[37] M. H. Park, J. Y. Park, H. J. Lee et al., "The novel PPAR $\alpha / \gamma$ dual agonist MHY 966 modulates UVB-induced skin inflammation by inhibiting NF- $\kappa$ B activity," PLoS ONE, vol. 8, no. 10, Article ID e76820, 2013.

[38] A. Mastrofrancesco, D. Kovacs, M. Sarra et al., "Preclinical studies of a specific PPAR $\gamma$ modulator in the control of skin inflammation," Journal of Investigative Dermatology, vol. 134, no. 4, pp. 1001-1011, 2014. 


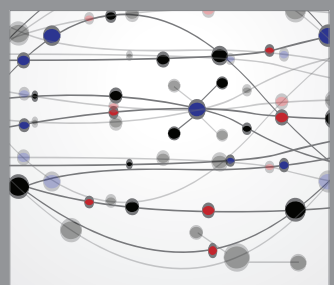

The Scientific World Journal
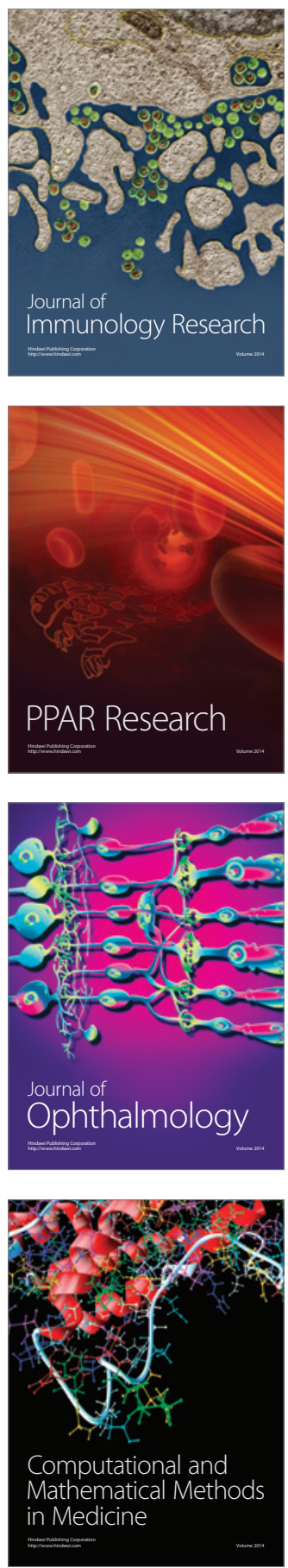

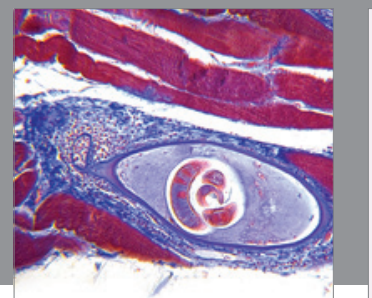

Gastroenterology

Research and Practice
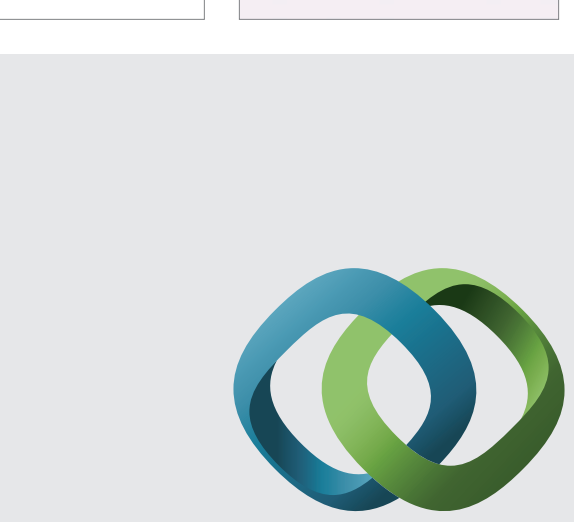

\section{Hindawi}

Submit your manuscripts at

http://www.hindawi.com
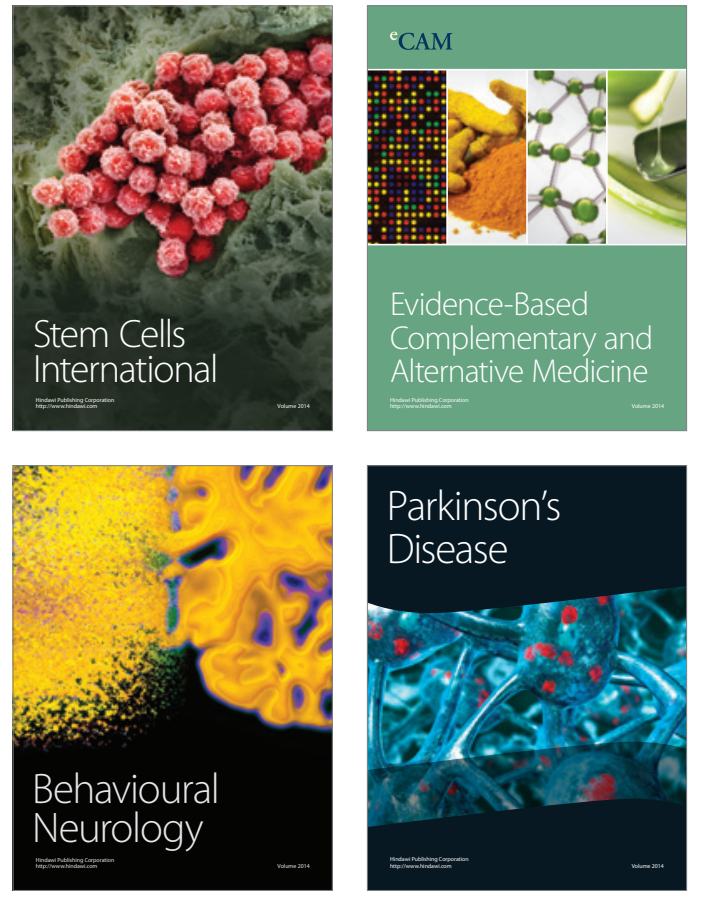
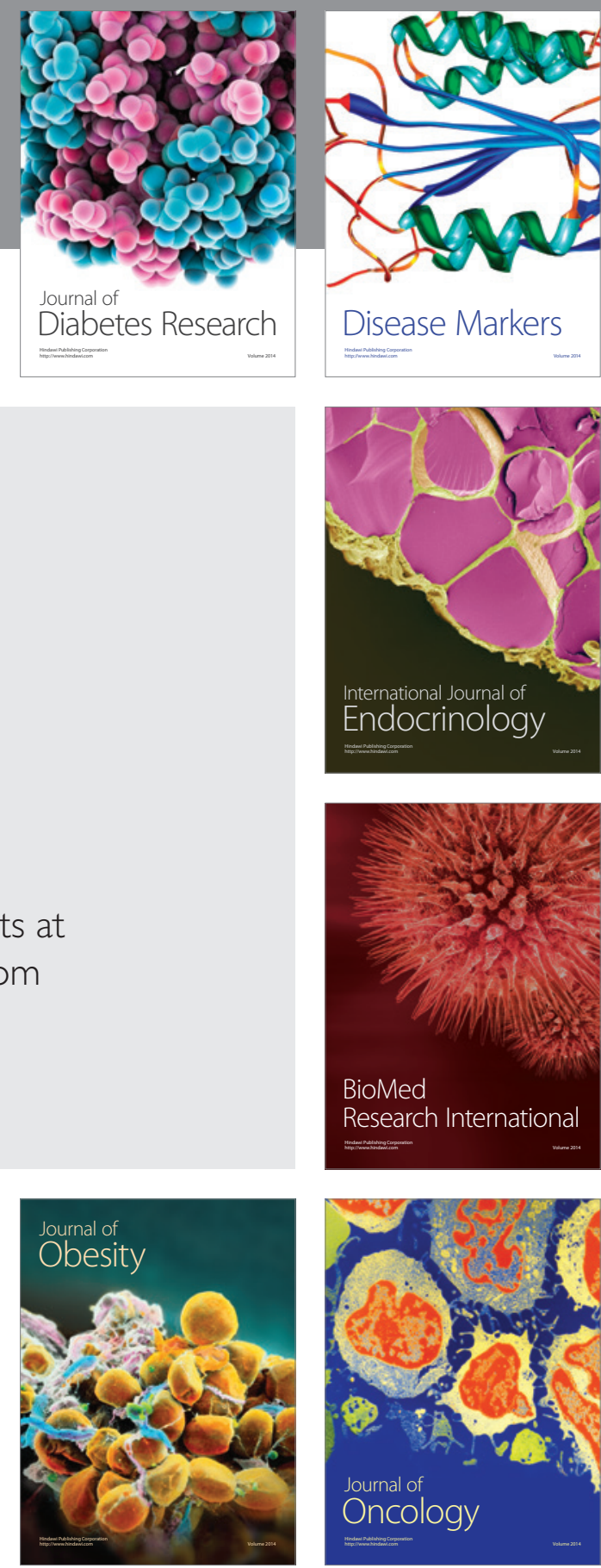

Disease Markers
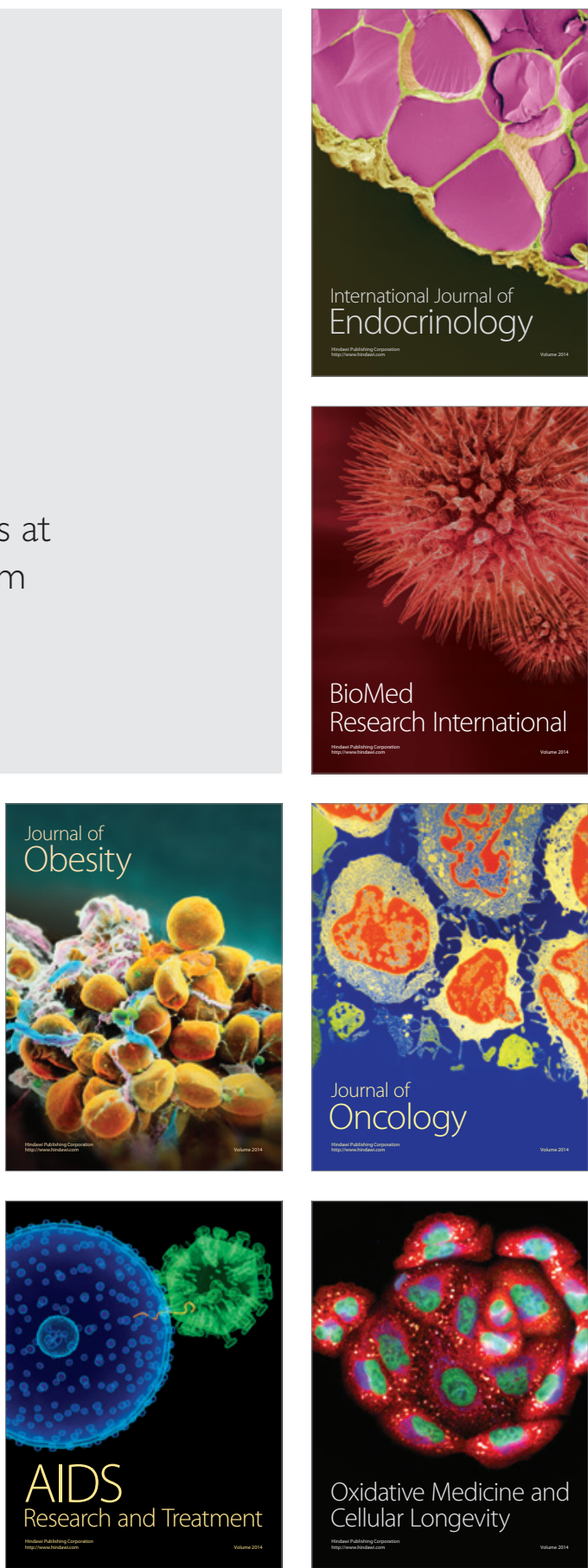